\title{
Estudiando el lenguaje y sus sentidos. Tradiciones y tendencias en las revistas latinoamericanas de la comunicación
}

Researching language and its meanings. Traditions in Latin American communication journals

ALEJANDRO BARRANQUERO

UNIVERSIDAD CARLOS III DE MADRID

ORCID: http://orcid.org/0000-0002-9264-9389

ADRIANA ANGEL

UNIVERSIDAD DE LA SABANA

ORCID: https://orcid.org/0000-0002-0667-0553

RESUMEN: El presente artículo examina la evolución y el estado actual de los estudios del lenguaje y sus sentidos en América Latina a partir de un análisis de contenido de 220 artículos publicados entre 2017 y 2019 en diez revistas latinoamericanas de referencia en el ámbito de la comunicación. Tras identificar cinco grandes tradiciones teóricas, los resultados evidencian el predominio de ciertos objetos, métodos y abordajes y demuestran que los estudios de discurso, semiótica, retóricas, narrativas y representación (no tanto la retórica) tienden a operar hoy de manera interconectada dada su orientación interdisciplinar y su vocación altamente teórica o ensayística, más que empírica. En el marco de la investigación comparada, América Latina se constituye como un locus autónomo de producción de conocimiento más allá de la fuerte presencia de enfoques eurocéntricos que mantienen a dicha producción algo alejada de la autonomía epistemológica que reclaman el giro decolonial y las epistemologías del sur.

Palabras clave: Retórica, discurso, narrativa, semiótica, representación, lenguaje, América Latina. 
ABSTRACT: This manuscript examines the evolution and current state of Latin American studies on language and its meanings departing from a content analysis of 220 articles published between 2017 and 2019 in ten flagship Latin American communication journals. After identifying five dominant theoretical traditions, the results show a set of predominant objects, methods and approaches, as well as demonstrate that studies on discourse, semiotics, rhetoric, narratives, and representation tend to operate interconnected today given their interdisciplinary, theoretical and essayistic orientation, rather than empirical. From a compared research perspective, Latin America constitutes itself as an autonomous locus of knowledge production despite the strong presence of Eurocentric approaches that keep such production away from the epistemological autonomy demanded by the decolonial turn and the epistemologies of the south.

Key words: Rhetoric, discourse, narrative, semiotics, representation, language, Latin America.

\section{INTRODUCCIÓN}

Históricamente, la investigación comunicacional en Latinoamérica se ha destacado por profundizar en un conjunto de temáticas que son fruto de los particulares contextos en los que se cimentó la disciplina durante los años 60 y 70: la comunicación alternativa y para el desarrollo, el análisis crítico de los mensajes mediáticos o la economía política de la comunicación (León-Duarte, 2001; Marques de Melo, 1998, 1999). En los años 90 irrumpieron con fuerza los Estudios Culturales y, más recientemente, objetos como la comunicación indígena o el impacto de Internet en las sociedades del conocimiento (Barranquero, Arcila y Arroyave, 2017). América Latina también ha sido un territorio fértil para estudios metateóricos que han analizado la comunicación como un campo diferenciado y transdisciplinar del conocimiento, que se nutre de distintas disciplinas y que, a su vez, propone una mirada transversal y comunicacional a los fenómenos sociales (Pereira, 2018; Vidales, 2015; Martín-Barbero, 2002; Vasallo de Lopes, 2000; LeónDuarte, 2001; Fuentes-Navarro, 1992). Más allá de esta dilatada trayectoria, sorprenden, sin embargo, los tímidos avances de la metainvestigación en el contexto latinoamericano, entendida esta como un método sistemático para ordenar los principales hallazgos teóricos de un área geográfica o disciplinar y de las propias metodologías que facilitan la consecución de dichos hallazgos (Piñeiro-Naval y Morais, 2019). El presente artículo se adscribe a dicha tradición para examinar uno de los campos con mayor raigambre histórica en el subcontinente: la comunicación entendida como un proceso lingüístico de construcción de significados y realidades mediáticas y sociales, que en adelante denominaremos estudios sobre el lenguaje y sus sentidos ${ }^{1}$.

Ya en su famoso De los Medios a las Mediaciones, Jesús Martín Barbero señaló que, desde los años 60, la investigación comunicacional latinoamericana se cimentó en base a dos tradiciones fundacionales que aún influyen en la manera de aproximarse a su

\footnotetext{
${ }^{1}$ Este artículo se enmarca en los proyectos I+D financiados "Transformaciones y Tendencias en los Estudios del Lenguaje en América Latina”, entre la Universidad de La Sabana y la Universidad Carlos III de Madrid y, asimismo, a "Mapas de la investigación en comunicación en las universidades españolas de 2007 a 2018” (ref. PGC2018-093358-B-I00), y dirigido por los Prof. Carmen Caffarel y Carlos Lozano.
} 
objeto de estudio: una primera, de corte funcionalista estadounidense y centrada en los efectos mediáticos, y una segunda, de orientación crítica europea, que se orientó a "descubrir y denunciar [...] las estratagemas mediante las cuales la ideología dominante penetra en [...] el mensaje produciendo determinados efectos" (Martín-Barbero, 1987: 221). A lo largo del tiempo, la tradición ideologicista, como la denominó Martín Barbero (1987), evolucionó en sus enfoques y énfasis, transitando desde los marcos sobreideologizados del primer estructuralismo (Lozano, 1996) hacia una diversificación e hibridación entre tradiciones críticas y otras de corte más positivista como los estudios de cultivo o de agenda-setting (León-Duarte, 2001:24). A una dirección parecida apuntan otros recuentos históricos sobre la investigación comunicacional en la región, que coinciden en señalar que los estudios sobre el mensaje o las ideologías han constituido una preocupación fundamental desde que pioneros como Eliseo Verón o Armand Mattelart dejasen su profunda huella en la construcción de este campo académico (Barranquero, Arcila y Arroyave, 2017; Moragas, 2011; León-Duarte, 2007; Torrico, 2004; Escudero, 1998; Marques de Melo, 1998; Fuentes Navarro).

Ya en el contexto anglosajón, metainvestigaciones recientes han demostrado la vitalidad de subcampos como la semiótica (Catt y Eicher-Catt, 2012), el lenguaje y la interacción social (Tracy y Haspel, 2004), la retórica (Bo-Wang, 2004) o el análisis del discurso (Bartesaghi y Pantelides, 2018), mientras que en España dos I+D de corte metaanalítico han puesto de manifiesto la preponderancia de la tradición contenudista; esto es, de los mensajes como objeto prioritario de investigación (Martínez-Nicolás y Saperas-Lapiedra, 2016) y de los métodos documentales -como el análisis de contenidocomo metodologías hegemónicas por encima de las experimentales o de intervención (Caffarel-Serra, Ortega-Mohedano y Gaitán-Moya, 2017: 223). Como señalamos, las metainvestigaciones sobre la comunicación en América Latina han sido, a todas luces, escasas, más allá de alguna notable excepción (Gómez-Palacio y Jara, 1989; Chaffee, Gómez-Palacio y Rogers, 1990; Piñeiro-Naval y Morais, 2019). No obstante, en tiempos recientes han aparecido los primeros recuentos sobre campos específicos como los estudios del periodismo (Mellado, 2012), las audiencias televisivas (Lozano y Frankenberg, 2008) o la comunicación para el desarrollo (Barranquero y Ángel, 2015). En el ámbito de los estudios del lenguaje y sus sentidos, los avances más significativos son reconstrucciones históricas y analíticas de la semiótica y la semiología (Romera Castillo, 2016; Scolari, 2011), impulsadas, en particular, desde sus principales centros de producción e influencia regional: Argentina (Zarowsky, 2008, 2017; Verón, 1974;), Chile (Parra Ortiz y Otazo Hermosilla, 2014; Parra, 2014; Gallardo y Sánchez, 1981) o Brasil (Pessoa de Barros, 2012). También en tiempos recientes se han publicado las primeras metainvestigaciones sobre el lenguaje y sus sentidos, profundizando en la recepción latinoamericana de las teorías de la agenda-setting (Gallego-Ramos, 2017), o en el estado del arte de los estudios del discurso (Rodrigo-Mendizábal, 2018), aunque ninguna de ellas desde la mirada integral y transdisciplinar que propone el siguiente artículo.

Con el objeto de paliar este déficit, este trabajo plantea una cartografía aproximativa de la historia y el estado actual del campo a partir de una revisión de artículos publicados en revistas paradigmáticas del ámbito de la comunicación. Dado que dicha tradición es demasiado extensa y heterogénea, la categorizamos bajo la denominación de estudios sobre el lenguaje y sus sentidos. Esta etiqueta nos ayuda a poner de manifiesto que el corpus que hemos analizado a modo de muestra no solo se pregunta por las ideologías o los significados dominantes en distintos textos (escritos, audiovisuales, etc.); sino que más bien se dirige a explorar los aspectos profundos en los que "se hace efectiva la comunicación misma", con lo que coincidimos con un 
metananálisis previo de Rodrigo-Mendizábal (2018: 17) acerca de los estudios del discurso.

El artículo también propone una categorización propia de las cinco tradiciones investigativas que dominan el área —estudios de retórica, discurso, narrativas, representación y semiótica - y se pregunta por otras cuestiones determinantes como los objetos y metodologías dominantes en los artículos analizados. Asimismo, indagamos en el grado de autonomía o dependencia epistemológica de dichas tradiciones y, en este sentido, hacemos nuestra la pregunta que hace unos años lanzaba Lucrecia Escudero (1998) al campo de la semiótica (¿existen los semiólogos latinomericanos?) para explorar la existencia de un subcampo relativamente autónomo dentro de la comunicación que explora el lenguaje y sus sentidos. Más allá del carácter aproximativo de las líneas que siguen, consideramos que mapas y reflexiones metaanalíticas como esta ayudan a detectar tendencias, lagunas y oportunidades de investigación (Cooper, Larry y Valentine, 2009; Romera-Castillo, 2016), además de fortalecer la madurez científica de una disciplina y sus áreas concretas de investigación (Piñuel y Morales, 2018).

\section{UNA REVISIÓN HISTÓRICA DE LOS ESTUDIOS DEL LENGUAJE EN AMÉRICA LATINA}

A mediados de los 70, la investigación comunicacional en América Latina comenzó a adquirir autonomía epistemológica y se constituyó como un espacio de bisagra o fusión entre dos tradiciones teóricas en conflicto (Beltrán, 1975): las teorías administradas o positivistas estadounidenses (y, en especial, el Funcionalismo) y los enfoques críticos europeos derivados de la Escuela de Frankfurt, el Estructuralismo y los Estudios Culturales. Desde una reinterpretación crítica de ambas tradiciones, Latinoamérica luchó contra la dependencia de los modelos importados para orientarse a la búsqueda de paradigmas autónomos y adaptados a las lógicas culturales del subcontinente (Beltrán, 1975, 1976). Ya en los tempranos 60, la semiótica estructuralista se había constituido como una de las primeras influencias teóricas, dialogando pronto con el marxismo y otras elaboraciones críticas regionales como las Teorías de la Dependencia o la Teología de la Liberación (Marques de Melo, Gobbi y Kunsch, 2002). En un contexto de luchas antiimperialistas y emancipadoras, la semiótica latinoamericana enfatizó en el análisis de problemáticas de corte político-social, un terreno en el que la región llegaría a adelantarse a Europa, tal y como reconocía Eliseo Verón en una de sus últimas entrevistas (Moragas, 2011: 187).

Deudora del influjo de centros europeos como el CECMAS en Francia o el Instituto Gemelli en Italia, la semiótica penetró con fuerza en países como Argentina, Chile o Brasil (Escudero, 1998) y sus primeros abordajes incorporaron una visión estructuralista atenta a las estructuras latentes en los mensajes (Torrico, 2004: 56-66) y que, por influencia del marxismo, acabó por entender los medios como aparatos ideológicos del estado (Althusser, 1974) o entes reproductores de intereses e ideologías de clase (Marx, 1867). En los 60, se publicaron obras de referencia en Argentina como Lenguaje y Comunicación Social (Verón, 1969), Mensajes y Señales (Prieto, 1967) o Happenings (Masotta et al., 1967), mientras que, en Chile, el gobierno de la Unidad Popular acogió a un núcleo crítico de investigadores en torno a la figura de Armand Mattelart, del que derivaron trabajos de referencia como los Cuadernos del CERÉN (ej. Mattelart, Mattelart y Piccini, 1970) o el popular Para leer al Pato Donald (Dorfman y Mattelart, 1971). En estos mismos países se editaron revistas pioneras como Comunicación y Cultura (1973-1985), impulsada por Héctor Schmucler y Armand Mattelart, o Lenguajes (1974-1980), dirigida por Eliseo Verón y principal órgano de la 
Asociación Argentina de Semiótica (Zarowsky, 2017; Moragas, 2011; Parra, 2014). Por su parte, en Brasil la Revista Brasileira de Semiótica Significação (1973-hoy) desempeñó un papel crucial en la divulgación de esta perspectiva (Pessoa de Barros, 2012), al tiempo que en otros países la semiótica se abría camino a estudios más cercanos a la retórica (Alcalde, 1996; Prieto Castillo, 1979) o al análisis narrativo de discursos e historias.

Hasta comienzos de los 80, muchos trabajos latinoamericanos - aunque no todos - estuvieron abocados al análisis de los mensajes de los medios masivos como soporte de la ideología dominante (Sunkel, 2006: 18). Pero la publicación de De los medios a las mediaciones (Martín-Barbero, 1987) y su propia relectura latinoamericana de la Escuela de Birmingham - Hall, Williams, Thompson - y de la tradición culturalista francesa - Bourdieu, De Certeau - allanaron el tránsito desde el enfoque ideologicista de los 70 a un interés más destacado por los estudios de recepción o por el carácter abierto del lenguaje y sus sentidos, del que Jesús González (1986) o Néstor García-Canclini (1982), entre otros, fueron destacados representantes. Desde finales de siglo XX, las investigaciones de Valerio Fuenzalida (1997), Elizabeth Lozano (2006) o Guillermo Orozco $(1991,1994)$ insistieron en las múltiples mediaciones y las decodificaciones inherentes en los procesos de recepción (Hall, 1980), con lo que ayudaron a percibir que los mensajes no son solo portadores de ideología, sino también de elementos de agencia, resistencia y transformación. Con el tiempo, la nueva orientación postestructuralista debilitó las antiguas posiciones estructurales, acusadas ahora de olvidar la historia, invisibilizar a los individuos, cosificar la estructura, matematizar el lenguaje, e incluso ideologizar en exceso los estudios de medios (Mattelart y Mattelart, 1997; Martín Barbero, 1987). Ya en las postrimerías del siglo XX, el denominado giro lingüístico (Rorty, 1992), y su interés por examinar cómo el lenguaje ejerce una determinación fundamental sobre la realidad (Jensen, 2015), terminó por desplazar la obsesión por el mensaje hacia el proceso mismo de la comunicación, entendiendo que el lenguaje no es un mero reflejo de la realidad, sino que construye y es construida por ella (Potter, 1996).

Desde esta última época, las investigaciones latinoamericanas sobre el lenguaje y sus sentidos se han diversificado hasta componer un prolífico campo transdisciplinar en el que conviven tradiciones diversas y en diálogo en su intento de comprender la complejidad de la comunicación. Conscientes de su amplitud y heterogeneidad, el presente artículo ha agrupado dichos estudios en torno a una categorización propia que sigue la senda de metainvestigaciones previas que ayudaron a sistematizar las diferentes perspectivas que conviven en la comunicación (Galindo-Cáceres, 2005; Mattelart y Mattelart, 1997). Nos referimos a modelos como el desarrollado por el estadounidense Robert Craig (1999), que identificó siete tradiciones en los estudios de comunicación: retórica, semiótica, fenomenológica, cibernética, sociopsicológica, sociocultural y crítica; o al programa latinoamericano Hacia una Comunicología posible (Gucom), que distinguió nueve fuentes históricas en el estudio de la comunicología: la sociología funcionalista, la sociología crítica, la sociología cultural, la sociología fenomenológica, la psicología social, la economía política, la cibernética, la lingüística y la semiótica (Galindo-Cáceres, 2005).

Este artículo identifica y resume cinco grandes tradiciones: (1) la semiótica, (2) la retórica —en las que coinciden Craig (1999) y el Gucom (Galindo Cáceres, 2005) (3) los estudios del discurso (4) las narrativas, (5) y las representaciones. Estas últimas perspectivas han cobrado fuerza en los últimos años según el recuento de RodrigoMendizábal (2018). En primer lugar, y heredera de reflexiones presocráticas y aristotélicas, la retórica intenta comprender el lenguaje como un arte del discurso, prestando especial atención a sus elementos persuasivos (Booth, 1988), sus figuras literarias (Alvarado, 2015), o sus modos de argumentación (Perelman y Olbrechts-Tyteca, 
1989) y de identificación textual (Burke, 1969). En segundo lugar, la semiótica ha tenido como objeto principal al lenguaje como portador de sentidos y a los modos de producción y recepción de signos y códigos, desde la semiología de origen europeo (Eco, 2000; Escudero, 1998) a la semiótica de corte estadounidense (Catt y Eicher-Catt, 2012; Barthes, 1999; Peirce, 1986). En tercer lugar, el estudio de los discursos se ha enfocado a analizar el lenguaje como práctica social en relación con los contextos y las relaciones de poder, influido, en buena medida, por la recepción de las obras de Foucault o van Dijk (Sawyer, 2002). En cuarto lugar, las narrativas se han centrado en el estudio de las estructuras y arquetipos que predominan en textos y relatos, inspiradas en trabajos como los de Greimas (1966) o Ricoeur (1984). Por último, los estudios de representación suelen retomar planteamientos de los Estudios Culturales (Hall, 1980, 1997) para estudiar la manera en que el lenguaje refleja y construye contenidos e identidades diversas.

\section{MARCO METODOLÓGICO}

Este artículo plantea una revisión de los estudios del lenguaje y sus sentidos en revistas latinoamericanas de referencia en el campo de la comunicación. Siguiendo los principios de la revisión documental (Staples y Niazi, 2007; Hart, 1998), el corpus comprende los artículos publicados entre 2017 y 2019 en relación con los estudios del lenguaje y sus cinco principales tradiciones; esto es, la retórica, la semiótica, los estudios del discurso, de las narrativas y de las representaciones. Las diez revistas que componen la muestra fueron seleccionadas de acuerdo a dos criterios. En primer lugar, se incluyeron publicaciones generalistas de acceso abierto indexadas en Scopus, considerada una base de datos de referencia para las revistas latinoamericanas y en español y cuyo sistema de selección es menos restrictivo que el de Web of Science (WoS), a cargo de la empresa Thomson Reuters (Aguado-López, Becerril-García, Arriola y Martínez-Domínguez, 2014). Nos referimos a: Palabra Clave, Comunicación y Sociedad, Chasqui, Comunicação, Mídia e Consumo, Intercom (hasta hace un año en Scopus) y Signo y Pensamiento. La muestra se completó con tres revistas de referencia no generales sino especializadas en las distintas tradiciones teóricas identificadas: Rétor (retórica), Galáxia (semiótica y hasta hace un año en Scopus) y Revista Latinoamericana de Estudios del Discurso. En segundo lugar, y para facilitar una perspectiva comparada interregional, se incluyó, al menos, una publicación por país y dos en el caso de aquellos países en los que se localizó una mayor producción editorial (Rodrigo-Mendizábal, 2018), optando siempre por la revista mejor indexada en Scopus. La Tabla 1 presenta el nombre y web de las revistas seleccionadas, su procedencia, su posición y área en Scopus, y la editorial u organización promotora. 
Tabla 1. Revistas recogidas en la muestra

\begin{tabular}{|c|c|c|c|}
\hline & \\
\hline & País & $\begin{array}{c}\text { Cuartil y Área } \\
\text { Scopus en } 2018\end{array}$ & $\begin{array}{c}\text { Editorial u organización } \\
\text { promotora }\end{array}$ \\
\hline Chasqui & Ecuador & $\begin{array}{l}\text { Q4 (Cultural } \\
\text { Studies) }\end{array}$ & $\begin{array}{c}\text { Centro Internacional de } \\
\text { Estudios Superiores de } \\
\text { Comunicación para América } \\
\text { Latina (CIESPAL) }\end{array}$ \\
\hline $\begin{array}{c}\text { Comunicação, } \\
\text { Mídia e Consumo }\end{array}$ & Brasil & $\begin{array}{c}\text { Q4 } \\
\text { (Communication) }\end{array}$ & $\begin{array}{c}\text { Escola Superior de } \\
\text { Propaganda e Marketing } \\
\text { (ESPM-SP) de São Paulo, } \\
\text { Brasil }\end{array}$ \\
\hline Intercom & Brasil & $\begin{array}{c}\text { Q4 } \\
\text { (Communication) }\end{array}$ & $\begin{array}{l}\text { Sociedade Brasileira de } \\
\text { Estudos Interdisciplinares } \\
\text { da Comunicação } \\
\text { (INTERCOM) }\end{array}$ \\
\hline Galáxia & Brasil & No consta & $\begin{array}{c}\text { Programa de Pós-Graduação } \\
\text { em Comunicação e } \\
\text { Semiótica }\end{array}$ \\
\hline $\begin{array}{c}\text { Comunicación y } \\
\text { Sociedad }\end{array}$ & México & $\begin{array}{c}\text { Q2 } \\
\text { (Communication) }\end{array}$ & Universidad de Guadalajara \\
\hline Cuadernos.info & Chile & $\begin{array}{c}\text { Q2 } \\
\text { (Communication) }\end{array}$ & $\begin{array}{l}\text { Pontificia Universidad } \\
\text { Católica de Chile }\end{array}$ \\
\hline Palabra Clave & Colombia & $\begin{array}{c}\text { Q3 } \\
\text { (Communication) }\end{array}$ & Universidad de La Sabana \\
\hline $\begin{array}{c}\text { Signo y } \\
\text { Pensamiento }\end{array}$ & Colombia & $\begin{array}{c}\text { Q4 } \\
\text { (Communication) }\end{array}$ & $\begin{array}{c}\text { Pontificia Universidad } \\
\text { Javeriana }\end{array}$ \\
\hline Rétor & Argentina & No consta & $\begin{array}{c}\text { Asociación Argentina de } \\
\text { Retórica }\end{array}$ \\
\hline $\begin{array}{c}\text { Revista } \\
\text { Latinoamericana } \\
\text { de Estudios del } \\
\text { Discurso }\end{array}$ & Argentina & No consta & $\begin{array}{c}\text { Asociación Latinoamericana } \\
\text { de Estudios del Discurso } \\
\text { (ALED) }\end{array}$ \\
\hline
\end{tabular}

Para la selección del corpus se leyeron los abstracts de todos los trabajos publicados entre 2017 y 2019. Esta lectura preliminar se orientó a filtrar aquellos artículos que cumpliesen con dos criterios: (1) tener por objeto de estudio la relación entre lenguaje, sentidos y producción de realidad, (2) y orientarse al análisis de fenómenos comunicacionales y culturales. Antes de aplicar el instrumento, se realizó un análisis inductivo de la mitad de las publicaciones con el fin de detectar el enfoque epistemológico predominante. Si bien la mezcla de perspectivas era patente en muchos de los artículos, se combinó la inducción con una aproximación deductiva a la literatura de referencia (Craig, 1999; Galindo Cáceres, 2005), que nos ayudó a construir una de las variables centrales para la selección y clasificación del corpus: la de las tradiciones teóricas dominantes. La Tabla 2 muestra el protocolo de análisis y, ya en Resultados, proporcionaremos una introducción a las variables más complejas. 
Tabla 2. Protocolo de análisis de contenido

\begin{tabular}{|c|c|}
\hline \multirow{2}{*}{ (n) } & \multirow{2}{*}{ Ítems } \\
\hline & \\
\hline Revista & $\begin{array}{l}\text { Chasqui, Palabra Clave, Comunicação, Mídia e Consumo, } \\
\text { Comunicación y Sociedad, Rétor, Galaxia, Cuadernos.info, etc. }\end{array}$ \\
\hline $\begin{array}{l}\text { Fenómeno } \\
\text { analizado }\end{array}$ & $\begin{array}{l}\text { Productos y procesos mediáticos tradicionales (prensa, } \\
\text { televisión, etc.), redes sociales y TIC, cine, publicidad, etc. }\end{array}$ \\
\hline $\begin{array}{l}\text { Referencia a otros } \\
\text { subcampos de la } \\
\text { comunicación }\end{array}$ & $\begin{array}{l}\text { Estudios de medios, comunicación política, comunicación y } \\
\text { género, comunicación para la salud, comunicación } \\
\text { organizacional, comunicación interpersonal, etc. }\end{array}$ \\
\hline $\begin{array}{l}\text { Ámbito geográfico } \\
\text { del fenómeno } \\
\text { objeto de estudio }\end{array}$ & $\begin{array}{c}\text { Norteamérica, América Latina, Europa, África, Australia, Asia, } \\
\text { o no especificado. }\end{array}$ \\
\hline $\begin{array}{l}\text { Naturaleza } \\
\text { metodológica }\end{array}$ & Cuantitativa, cualitativa, crítica, conceptual, mixta \\
\hline $\begin{array}{l}\text { Tamaño del } \\
\text { corpus o muestra }\end{array}$ & $1,2,3-20$, más de 20 , no especificado \\
\hline $\begin{array}{l}\text { Tipo de escritura } \\
\text { académica }\end{array}$ & Clásica, alternativa \\
\hline $\begin{array}{c}\text { Técnica } \\
\text { dominante de } \\
\text { investigación o } \\
\text { interpretación de } \\
\text { los datos }\end{array}$ & $\begin{array}{c}\text { Análisis retórico, de discurso, narrativo, semiótico, de } \\
\text { representación }\end{array}$ \\
\hline $\begin{array}{l}\text { Tradición teórica } \\
\text { predominante }\end{array}$ & Retórica, discurso, narrativa, representación, semiótica \\
\hline $\begin{array}{l}\text { Autores de } \\
\text { referencia }\end{array}$ & $\begin{array}{c}\text { Autores de referencia europeos, norteamericanos, o } \\
\text { latinoamericanos }\end{array}$ \\
\hline
\end{tabular}

\section{RESULTADOS}

\subsection{TEMÁTICOS, ÁMBITOS GEOGRÁFICOS Y METODOLOGÍAS}

En las revistas analizadas se localizaron un total de 220 artículos sobre retórica, semiótica, narrativas, discursos y representaciones, distribuidos de la siguiente manera: Galáxia (41), Chasqui (33), Revista Latinoamericana de Estudios del Discurso (32), Retor (25), Palabra Clave (19), Comunicação, Mídia e Consumo (17), Comunicación y Sociedad (16), Cuadernos.info (13), Intercom (13) y Signo y Pensamiento (11).

Domina en ellos el análisis de productos y procesos mediáticos masivos en tradicionales - como noticias en prensa, programas de televisión, etc. - (69) y, en menor medida, redes sociales y otros productos infotecnológicos (20). Destaca asimismo el análisis fílmico (23), que separamos del resto de medios y que denota una profunda influencia de los estudios del lenguaje en la constitución del campo de los film studies. En ocasiones también se examinan los discursos de figuras políticas representativas (14) 
y llas campañas publicitarias (14). También es evidente la intersección de los estudios del lenguaje con subcampos como los estudios de los medios en general (69 artículos) y la comunicación política (44). Más minoritarios son los trabajos que exploran la comunicación y el género (22), la comunicación intercultural (18), la comunicación de la salud (11), la religiosa (5), la organizacional (5) y la interpersonal (4).

En relación con el lugar donde se desarrolla el fenómeno analizado existe un interés predominante por temáticas latinoamericanas (133 artículos), seguida a bastante distancia por fenómenos europeos (31 artículos, referidos principalmente a España) y norteamericanos (15, sobre todo, a EE.UU.). Es importante señalar que la naturaleza teórica de algunos artículos hace que estos no se centren en ninguna región en particular, sino que más bien refieran al propio campo académico de los estudios del lenguaje al analizar, por ejemplo, una teoría, tradición, obra o autor. La Gráfica 1 evidencia la prioridad de las temáticas latinoamericanas, a la vez que cierto grado de apertura e internacionalización.

\section{Gráfica 1. Ámbito geográfico de los fenómenos estudiados}

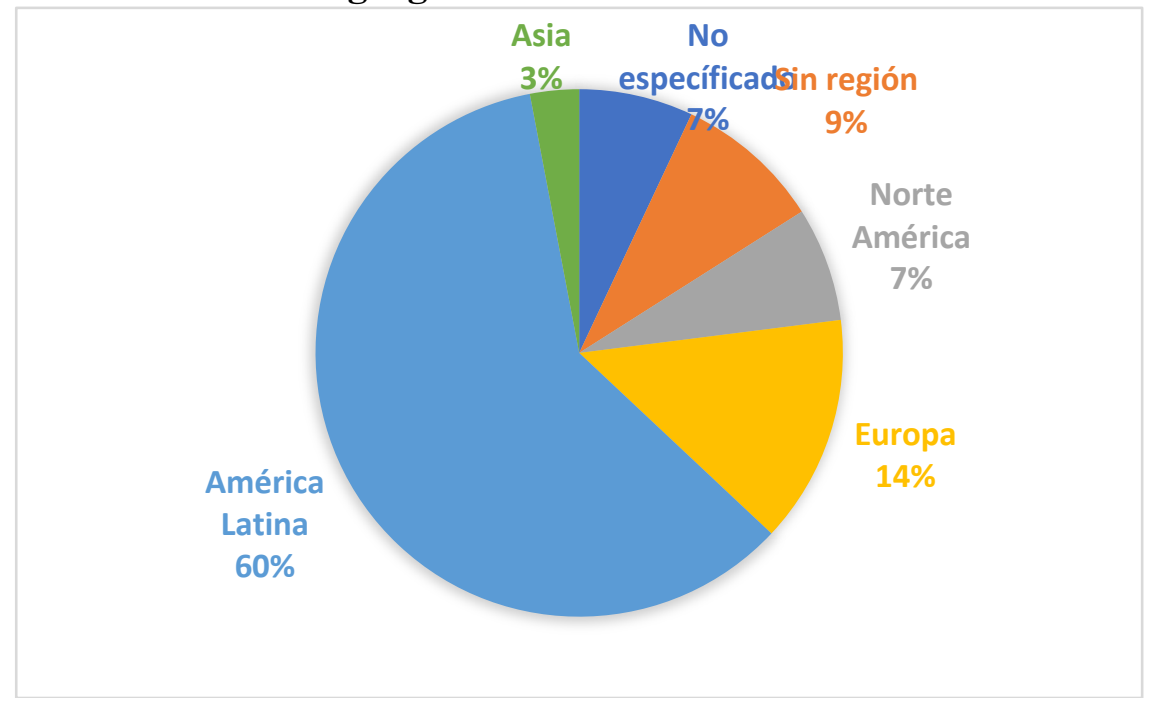

En su naturaleza metodológica, casi la mitad de los artículos (108) reflexiona sobre la manera en la que el lenguaje opera en relación con un fenómeno de estudio - de tipo mediático, de comunicación política, etc. - desde teorías y enfoques críticos como la semiótica, el estructuralismo o el postestructuralismo, que, a su vez, constituyen su principal sustento metodológico (Merrigan y Huston, 2009). El propósito de estas perspectivas críticas consiste además en identificar las relaciones entre lenguaje y poder $\mathrm{y}$, específicamente, el rol del lenguaje (visto como signos, discursos, representaciones, narrativas o retóricas) en la creación, reproducción, jerarquización, representación o resistencia frente a estructuras y relaciones de poder inmersas en contextos socioculturales y políticos particulares (Alexander y Potter, 2001).

El número significativo de estudios críticos se relaciona con el tamaño del corpus analizado, que, en su mayor parte, está conformado por 1 o 2 casos (en 50 y 39 artículos respectivamente), lo que demuestra que muchos trabajos se centran en realidad en el estudio de un discurso político, una pieza mediática, una obra cinematográfica o un evento comunicativo. Tras las metodologías críticas, se sitúan los estudios elaborados con métodos cualitativos (57) y con corpus que abarcan más de 3 elementos. En un sentido opuesto, solo 29 artículos se orientan al estudio de más de 20 casos o unidades de análisis, la mayoría recurriendo a métodos cuantitativos como el análisis de contenido, que es una técnica predominante en 15 artículos. También sobresale la presencia de los trabajos que 
denominamos de corte conceptual (36); esto es, que no se basan en procedimientos empíricos ni examinan objetos de estudio, sino que más bien ofrecen una discusión conceptual acerca de un aspecto específico del lenguaje y sus sentidos. La Gráfica 2 describe la naturaleza metodológica predominante en los manuscritos analizados.

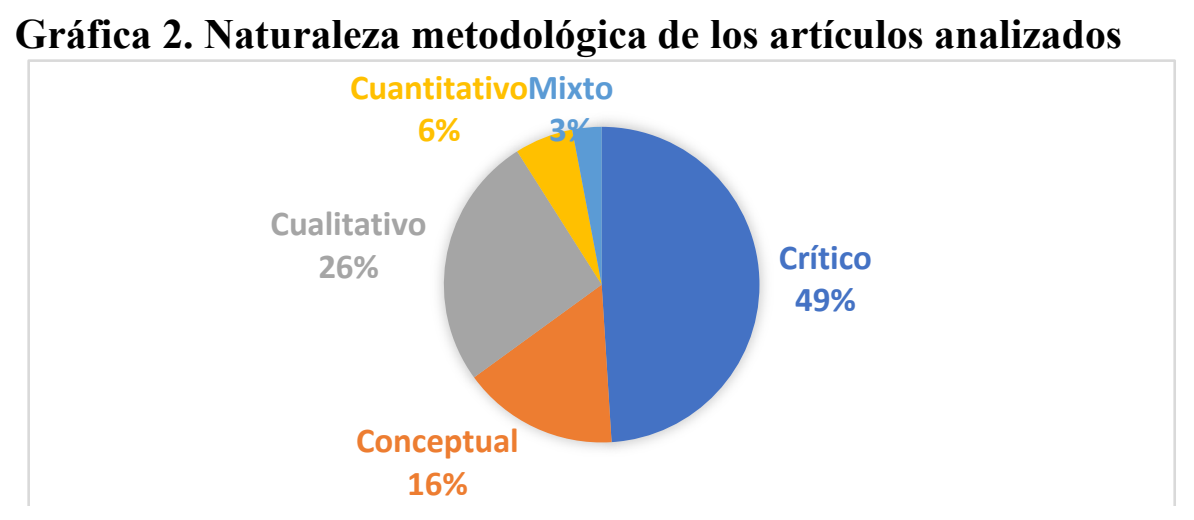

El alto número de los artículos que adoptan una metodología crítica explica que la mayoría sigue lo que denominamos una estructura alternativa de escritura científica (131); es decir, que no se guían por la organización estandarizada habitual de los artículos de corte empírico, que está basada en una introducción y marco teórico, metodologías, resultados y conclusiones. En menor medida, 89 artículos se acogen a la estructura clásica o estandarizada de la escritura científica, más propia de la investigación comunicacional de corte psicológico y sociológico, e incluso requerida en las normas de publicación de muchas revistas.

En relación con las técnicas de investigación empleadas, la mayoría de los artículos no especifican ningún método (64), sino que más bien ofrecen una interpretación de tipo teórico o conceptual acerca de cómo opera el lenguaje con relación distintos objetos de estudio. Este dato se relaciona tanto con la escritura alternativa que domina en muchos trabajos $-\mathrm{y}$ en la que no se exige situar una sección explícitamente metodológica - como con la amplia variedad de ensayos. En los casos en que sí se explicita la técnica, predomina el análisis de discurso (56), seguido a bastante distancia del análisis semiótico (19), de contenido (15) y narrativo (13), tal y como refleja la Gráfica 3 . Esta variable demuestra una clara preferencia por los métodos críticos de corte europeo (semiótica, narrativa, análisis crítico de discurso), por encima de perspectivas cuantitativas de origen estadounidense como el análisis de contenido.

\section{Gráfica 3. Técnicas de investigación o interpretación de datos}

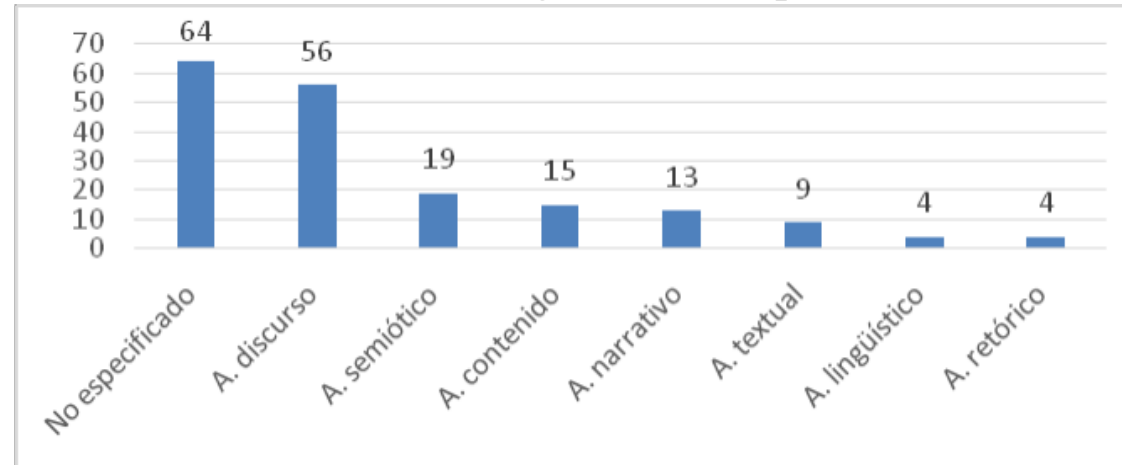




\subsection{TRADICIONES TEÓRICAS Y AUTORES DE REFERENCIA}

Con relación a la tradición investigativa, los artículos se inscriben, sobre todo, en los estudios de representación (68 artículos) y de discursos (60), seguidos a cierta distancia por los trabajos de retórica (34), narrativas (30) y semiótica (28).

\section{Gráfica 4. Tradición teórica a la que se adscribe el artículo}

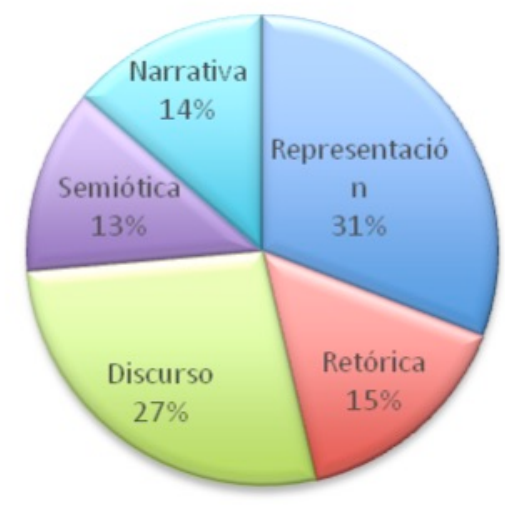

Los manuscritos que abordan la retórica (34) se adscriben en sus dos terceras partes (25) a la revista especializada Rétor. En comparación con el resto de tradiciones definidas - semiótica, representaciones, etc.-, la retórica no dialoga de manera significativa con el resto de perspectivas, sino que adolece de cierto ensimismamiento y enfoque autorreferencial. A diferencia de estudios homólogos en EE.UU., la tradición retórica latinoamericana no se adscribe tan claramente al campo de la comunicación, algo evidente en el hecho de que las principales asociaciones científicas latinoamericanas (como la Asociación Latinoamericana de Estudios de la Comunicación-ALAIC o la Federación Latinoamericana de Facultades de Comunicación Social-FELAFACS) no cuentan con secciones especializadas en la materia. Asimismo, las restantes cuatro tradiciones - discursos, signos, narrativas y representaciones - tienden a imbricarse al proponer una lectura holística o ciertas aproximaciones al lenguaje y sus sentidos, y más allá de que cada tradición conserve ciertos énfasis y objetos tradicionales.

A excepción de la retórica, en América Latina las restantes cuatro tradiciones discursos, signos, narrativas y representaciones - parecen definirse en términos de otras. Por ejemplo, aunque el núcleo principal de los estudios del discurso sea el uso del lenguaje en relación con el poder y los contextos sociales, sus trabajos suelen recurrir a categorías de otras tradiciones para acabar definiendo el discurso como sistemas de signos (noción más cercana a la semiótica), depositario de representaciones, o incluso portador de narrativas. Igualmente, otros artículos conciben la representación como una construcción discursiva; la semiótica como estudios del significado; o la narrativa como expresión discursiva de la identidad. Buen ejemplo de ello es el trabajo de Martyniuk y Silva (2018), que inscribe las narrativas mercadológicas en la tradición semiótica, aunque finalmente acaba realizando un estudio narrativo con objeto de demostrar la manera en que las estrategias publicitarias se estructuran en el escenario de la comunicación en red. Por su parte, Alvarado y Peralta (2017) hacen dialogar retórica, hermenéutica y semiótica a fin de comprender la forma en que el lenguaje ha privilegiado ciertos modos retóricos en distintos momentos de su historia. Con estos ejemplos, no queremos indicar que en los artículos se perciban incoherencias teóricas, sino más bien que las fronteras entre dichas tradiciones no están tan marcadas, ni son en exceso determinantes a la hora de acotar teóricamente los objetos. 
El desdibujamiento de estos límites tiene como correlato un cierto solapamiento metodológico, dado que las distintas técnicas de investigación o interpretación descritas en el apartado anterior no se emplean de manera pura o aislada, sino que más bien se apoyan unas en otras de cara a analizar cómo opera el lenguaje en relación con un objeto de estudio determinado. Por ejemplo, aunque el trabajo de Vizcarrondo (2017) sobre el discurso de cuatro periódicos estadounidenses acerca del debate sobre la ley SB1070 de Arizona se inscribe en la tradición de análisis de discurso, la autora emplea una metodología semiótica para dar cuenta de los contenidos de los debates que rodearon a esta ley de inmigración. Por su parte, Campetella (2017) inscribe en la tradición retórica su investigación sobre la designación de la ciudad de Bahía Blanca (Argentina) como polo de desarrollo regional, pero su estudio finalmente se lleva a cabo apoyado en el análisis de discurso.

En otro orden de cosas, el análisis conceptual demuestra que los artículos de las cinco tradiciones suelen apoyarse en una amplia diversidad de marcos teóricos y académicos de referencia, entre los que no predomina ninguno de manera evidente, sino más bien abordajes teóricos interconectados. No obstante, hay un conjunto de nombres que se repiten como base teórica de muchos artículos: la mayoría de procedencia europea (Michel Foucault, Paul Ricoeur, Teun van Dijk, Quintiliano, etc.) o estadounidense (Judith Buttler, Norman Fairclough, Charles Peirce, etc.) y, en menor medida, latinoamericana (sobre todo, Neyla Pardo y Eliseo Verón).

Aunque el abanico teórico es amplio, las perspectivas más adoptadas son los estudios críticos del discurso, la retórica neoaristotélica, el postestructuralismo, los estudios culturales y las perspectivas feministas. De hecho, cada una de las tradiciones delimitadas suele operar citando a un conjunto de autores de cabecera. Por ejemplo, los estudios retóricos se basan predominantemente en las perspectivas clásicas y neoaristotélicas representadas por Aristóteles, Cicerón y Quintiliano, en tanto los de discurso se fundamentan en las teorías críticas de Teun van Dijk o Norman Fairclough. Los estudios de representación suelen apelar a la tradición de los Estudios Culturales y a planteamientos como los de Michel Foucault, mientras que en los narrativos priman referencias como Paul Ricoeur o Algirdas Julien Greimas. Finalmente, los semióticos se soportan en posiciones clásicas y pragmáticas en las que Charles Pierce es nombrado con frecuencia.

Entre las referencias más citadas, llama la atención la escasa presencia de nombres latinoamericanos. A modo de prueba, en nuestros 220 artículos optamos por contabilizar quiénes eran los autores latinoamericanos más representativos tomando como referencia los nombres detectados por Rodrigo-Mendizábal (2018) en el ámbito del análisis de discurso. Entre los señalados por él, en nuestro corpus solo se citaban, y con escasa frecuencia, académicos/as como Eliseo Verón (12 referencias en 220 artículos), Neyla Pardo (10), Jesús Martín Barbero (9), Elvira Narvaja de Arnoux (8) y, a más distancia, Beatriz Sarlo, Adriana Bolívar y Armand Mattelart (3 menciones). Otros autores/as representativos de dicha tradición como Jorge Lazarte, Freda Indursky, Evandra Grigoletto, Carlos Piovezzani, Daniel Mendoza, Óscar Iván Londoño, Gladys Lucía Acosta, Jorge Quesada, Teresa Carbó, Daniel Prieto Castillo o Iván Rodrigo-Mendizábal no fueron citados ni una sola vez en la muestra escogida.

En comparación con las referencias foráneas, cabe señalar entonces que los estudios latinoamericanos sobre el lenguaje y sus sentidos se basan mayoritariamente en referencias a autores europeos, entre los que destacan, sobre todo, Teun van Dijk, Michael Foucault y Norman Fairclough, con 30 citas cada uno. Este dato llama la atención si tenemos en cuenta el llamado reciente a decolonizar las ciencias sociales latinoamericanas y a revitalizar las epistemologías del Sur, planteado por el Programa 
Modernidad/Colonialidad (Escobar, 2010; Mignolo, 2007), además de por otras perspectivas decoloniales en clave feminista, intercultural o indígena. Por otra parte, y aunque el giro decolonial ha comenzado a permear campos comunicacionales como la comunicación y el desarrollo o la comunicación comunitaria (Barranquero, Arcila y Arroyave, 2017; Barranquero y Ángel, 2015), este no es tan evidente en los estudios del lenguaje, a pesar de que la teoría latinoamericana de la comunicación se construyó, en buena medida, desde la confrontación y/o readaptación de perspectivas foráneas (Beltrán, 2014), tales como los estudios semióticos estructuralistas de los años 60 que ya comentamos (Martín Barbero, 1987).

\section{CONCLUSIONES}

El presente artículo ha explorado las temáticas, enfoques y metodologías dominantes en los artículos comunicacionales relacionados con el lenguaje y sus sentidos. Los resultados evidencian que América Latina ha transitado paulatinamente desde una perspectiva analítica ideologicista —o más preocupada por "desmontar los mitos y develar la ideología oculta de los mensajes" (Scolari, 2011: 15) — hacia una de corte postestructuralista, que entiende la comunicación como un proceso complejo que va más allá de la búsqueda de las estructuras fijas de significado. De hecho, la mayor parte de los artículos analizados tiende a rechazar la idea de que el lenguaje opera de manera matemática reflejando sentidos fijos subproducto de otros determinantes (infraestructurales, políticos, etc.), o de forma referencial limitándose a representar la realidad de manera transparente (Potter, 1996).

El estudio del corpus también permite inferir la progresiva confluencia teórica y metodológica de un conjunto de tradiciones que en sus inicios fueron más disímiles pero que, con el paso del tiempo, han tendido a converger en sus enfoques y métodos en el momento de analizar los sentidos de la comunicación. En la lógica del campo científico de Bourdieu (2003), podemos afirmar que el desvanecimiento de los límites entre los estudios de discursos, narrativas, representaciones y signos $-\mathrm{y}$, en menor medida, retórica - demuestra una tensión entre la transdisciplinariedad a la que apuntan los estudios de la comunicación (Vidales, 2015) y la disciplinariedad que reproducen los departamentos y asociaciones latinoamericanas sobre estudios del lenguaje (ej. asociaciones de semiótica, retórica, etc.). Estas estructuras operan en conjunto con asociaciones profesionales y revistas científicas hasta determinar las lógicas y reglas de producción, circulación y uso del conocimiento (Osborne, 2015), que, aunque a veces arbitrarias, no son neutrales en la medida en que definen qué es lo que cuenta como conocimiento válido y cómo organizarlo, además de legitimar y preponderar áreas, teorías, autores, metodologías o planes de estudio.

Por su parte, el análisis metodológico desvela el dominio de trabajos de corte crítico, cualitativo, conceptual, teórico y metateórico que se alejan bastante de la influencia proyectada por la psicología o la sociología en el campo de la comunicación. En esta línea, buena parte de los artículos analizados posee un estilo de escritura alternativo con respecto al modelo estandarizado dominante en el campo de la comunicación (Goyanes, 2017). En el ámbito de la escritura científica, este consiste, entre otros rasgos, en una estructura narrativa tipo IMRaD (Introducción, Métodos, Resultados y Discusión), que domina en el campo anglosajón desde finales de siglo XX (Sollaci y Pereira, 2004) y que fue importada desde el campo de las ciencias experimentales (Bazerman, 1988).

En otro orden de cosas, los estudios latinoamericanos del lenguaje se constituyen como un locus propio de enunciación por cuanto producen abundante conocimiento sobre 
fenómenos locales y regionales (Escudero, 1998). No obstante, el campo sigue siendo un espacio altamente dependiente de influencias foráneas, de las que dan buena cuenta las autorías referenciadas y la escasa presencia de nombres latinoamericanos. Así, y aunque detectamos abundantes artículos que intentan "hacer 'hablar' al discurso desde adentro" (Rodrigo-Mendizábal, 2018: 64), aún no se aprecia ni un auténtico diálogo interregional entre latinoamericanos y europeos, ni una búsqueda explícita de una perspectiva epistemológica autónoma latinoamericana, en el sentido que hoy se percibe en ámbitos como la comunicación y el desarrollo (Contreras, 2016; Restrepo y Valencia, 2017), o los estudios que reivindican la afrocentricidad (Asante y Miike, 2013) o la asiacentricidad (Miike, 2010) en el ámbito de la comunicación. Dicha perspectiva profundizaría en la tarea de descolonización propuesta por el Programa Modernidad/Colonialidad y otras perspectivas críticas contemporáneas.

Rechazando la idea de una producción latinoamericana homogénea 0 estandarizada, el estudio confirma que los estudios del lenguaje y sus sentidos en América Latina son un subcampo comunicacional diverso que aborda muy distintos fenómenos a partir de la yuxtaposición de distintas tradiciones teóricas y metodologías. El subcampo refleja, a su vez, las características que Marques de Melo (1999) atribuyó a la investigación en comunicación en Latinoamérica, en especial el hibridismo metodológico, la dimensión extranacional y el compromiso ético-político. Sin embargo, aún no se profundizado lo suficiente en la línea del auténtico mestizaje teórico descrito por el autor; es decir, en una combinación de perspectivas y autores latinoamericanos con trabajos de otras latitudes. Este hecho le permitiría alejarse de las miradas eurocéntricas tantas veces cuestionadas por los estudios decoloniales.

Entre las potencialidades de esta investigación, destacamos la propia distinción de las cinco tradiciones, que puede contribuir a la formulación de estudios comparados a nivel estatal o entre culturas investigativas diversas (Hepp y Couldry, 2009) como la latinoamericana o la anglosajona. No obstante, esta categorización de tradiciones no ha pretendido homogeneizarlas ni reducirlas a una sola macroescuela - la de los estudios del lenguaje y sus sentidos-, sino más bien operacionalizarlas de cara a distinguir diferentes énfasis teóricos y metodológicos en el abordaje de las relaciones entre lenguaje y la interacción social en distintos contextos comunicativos. Entre las limitaciones, el artículo ha analizado exclusivamente revistas indexadas en Scopus, pero este mismo esfuerzo podría extrapolarse a otras bases de datos - JCR/WoS, Dialnet, Latindex - , o incluso al propio campo de los libros y monografías, que históricamente se constituía como el formato de divulgación más habitual en el área.

\section{REFERENCIAS BIBLIOGRÁFICAS}

Aguado-López, Eduardo, Becerril-García, Arianna., Arriola, Miguel, y MartínezDomínguez, Néstor. 2014. «Iberoamérica en la ciencia de corriente principal (Thomson Reuters/Scopus): una región fragmentada». Interciencia, 39(8): 570579.

Alexander, Alison y Potter, James. 2001. How to publish your communication research: An insider's guide. Thousand Oaks, CA: Sage.

Althusser, Louis. 1974. Ideología y aparato ideológicos de Estado. Buenos Aires: Nueva Visión.

Alvarado, Carlos. 2015. «El giro retórico. Las derivas textuales en el cine perverso de Alex de la Iglesia». Escribanía, 10(1): 83-102. 
Alvarado, Carlos y Peralta, Misael. 2017. «Alegoría y símbolo en el cine barroco y moderno: Estrategias retóricas, amplificación de sentido». Galaxia, 36: 18-30. doi: https://doi.org/10.1590/1982-2554233276

Asante, Molefi y Miike, Yoshitaka. 2013. «Paradigmatic issues in intercultural communication studies: An afrocentric-asiacentric dialogue». China Media Research, 9(3): 1-19. Disponible en: https://go.gale.com/ps/anonymous?id=GALE\%7CA340944536\&sid=googleSchol $\operatorname{ar} \& v=2.1 \& i t=r \&$ linkaccess $=$ abs\&issn $=1556889 X \& p=A O N E \& s w=w$

Barranquero, Alejandro y Ángel, Adriana M. 2015. «La producción académica sobre Comunicación, Desarrollo y Cambio Social en las revistas científicas de América Latina». Signo $\quad$ S $\quad$ Pensamiento, 34(67), 30-58. https://doi.org/10.11144/Javeriana.syp34-67.pacd

Barranquero, Alejandro, Arcila, Carlos y Arroyave, Jesús. 2017. Manual de Teoría de la Comunicación II. Pensamientos Latinoamericanos. Barranquilla: Universidad del Norte y Ediciones de la U.

Bartesaghi, Marialena y Pantelides, Kate. 2018. «Why critique should not run out of steam: a proposal for the critical study of discourse». Review of Communication, 18(3): 158-177. doi: https://doi.org/10.1080/15358593.2018.1479884

Barthes, Roland. 1999. Mitologías. Buenos Aires: Siglo XXI.

Bazerman, Charles. 1988. Shaping written knowledge: The genre and activity of the experimental article in science. Madison: University of Wisconsin Pres.

Beltrán, Luis Ramiro. 1975. «Research ideologies in conflict. Forms of cultural dependency». Journal of Communication, 25(2): 187-193. Disponible en: https://idl-bnc-idrc.dspacedirect.org/handle/10625/20010

Beltrán, Luis Ramiro. 1976. «Alien premises, objects and methods in Latin American Communication Research». Communication Research, 3(2): 107-134.

Beltrán, Luis Ramiro. 2014. Comunicología de la liberación, desarrollismo y políticas públicas. Málaga: Luces del Gálibo.

Booth, Wayne. 1988. The company we keep: An ethics of fiction. Berkeley, CA: University of California Press.

Bourdieu, Pierre. 2003. Cuestiones de sociología. Madrid: Itsmo.

Burke, Kenneth. 1969. A rhetoric of motives. Berkeley, CA: University of California Press.

Caffarel, Carmen, Gaitán, Juan, Lozano, Carlos y Piñuel, José. 2018. Tendencias metodológicas en la investigación académica sobre Comunicación. Salamanca: Comunicación Social.

Caffarel-Serra, Carmen, Ortega-Mohedano, Félix y Gaitán-Moya, Juan Antonio. 2017. «Investigación en comunicación en la universidad española en el período 20072014». El Profesional de la Información-EPI, 26(2), 218-227. doi: 10.3145/epi.2017.mar.08

Campetella, Luciano. 2017. «Tras las huellas de 'Bahía Blanca polo de desarrollo': Contribución al análisis de una memoria retórico argumental». Rétor, 7(1): 1-20. Disponible en: https://dialnet.unirioja.es/servlet/articulo?codigo $=7008407$

Casado, Miguel y Fernández-Quijada, David. 2013. «El estado de la investigación española en políticas de comunicación: una revisión bibliométrica (2002-2011)». Trípodos, 32: 113-132.

Catt, Isaac y Eicher-Catt, Deborah. 2012. «Semiotics in mainstream American communication studies: A review of principal U.S.A. journals in the context of communicology». Review of Communication, 12(3): 176-200. doi: $10.1080 / 15358593.2012 .666260$ 
Contreras, Adalid. 2016. «Aruskipasipxañanakasakipunirakispawa». En Franscisco Sierra y Claudio Maldonado (Coords.). Comunicación, Decolonialidad y Buen Vivir. Quito: CIESPAL, 59-94.

Cooper, Harry M., Hedges, Larry V. y Valentine, Jeffrey C. (eds.). 2009. The handbook of research synthesis and meta-analysis. New York: Russel Sage Foundation.

Craig. Robert. 1999. «Communication theory as a field». Communication Theory, 9: $119-161$.

Dorfman, Ariel y Mattelart, Armand. 1971. Para leer al Pato Donald. Comunicación de masas y colonialismo. Valparaíso: Ediciones Universitarias de Valparaíso. $1^{\text {a }}$ Ed.

Eco, Umberto. 2000. Tratado de semiótica general. Barcelona: Lumen.

Escobar, Arturo. 2010. "Worlds and Knowledges Otherwise: the Latin American modernity/coloniality research program». En Walter Mignolo y Arturo Escobar (Eds.). Globalization and the Decolonial Option. New York: Routledge, 33-48.

Escudero, Lucrecia. 1998. «La Federación Latinoamericana de Semiótica: ¿existen los semiólogos latinoamericanos?». Signa: Revista de la Asociación Española de Semiótica, 7: 17-36. Disponible en: https://dialnet.unirioja.es/servlet/ articulo?codigo $=176511$

Fuentes-Navarro, Raúl. 1992. Un campo cargado de futuro. El estudio de la comunicación en América Latina. México: FELAFACS.

Galindo-Cáceres, José. 2005. Hacia una Comunicología posible. San Luis de Potosí: Universidad Autónoma de San Luis Potosí.

Gallardo, Andrés y Sánchez, Jorge. 1981. «Semiotics in Chile». En Tomas Sebeok y Jean Umiker-Sebeok (eds.), The semiotic Sphere. New York: Springer, 99-110.

Gallego-Ramos, José. 2017. «Existe... pero no se ve: investigación de Agenda Setting en América Latina». La Trama de la Comunicación, 2(2): 87-108. Disponible en: https://www.redalyc.org/jatsRepo/3239/323952120005/html/index.html

García-Canclini, Néstor. 1982. Las culturas populares en el capitalismo. México: Nueva Imagen.

Gómez-Palacio, Carlos, Chaffee, Steven y Rogers, Everett M. 1990. «Mass communication research in Latin America: Views from here and there». Journalism \& Mass Communication Quarterly, 67(4), 1015-1024. doi: 10.1177/107769909006700402

Gómez-Rodríguez, Gabriela, Morrel, Arley y Gallo-Estrada, Cristina. 2017. «A 30 años de Comunicación y Sociedad: cambios y permanencias en el campo académico de la comunicación». Comunicación y Sociedad, 30: 17-44. Disponible en http://www.scielo.org.mx/scielo.php?script=sci_arttext\&pid=S0188252X2017000300017

González, Jorge. 1986. Cultura(s). México: Universidad de Colima.

Goyanes, Manuel. 2017. Desafío a la investigación estándar en comunicación. Crítica y alternativas. Barcelona: UOC.

Greimas, Algirdas. 1966. Sémantique structurale: recherche de méthode. Paris: Larousse.

Hall, Stuart. 1980. «Encoding/Decoding». En S. Hall, D. Hobson, A. Lowe y P. Willis (eds.), Culture, Media, Language: Working Papers in Cultural Studies. Birmingham: Hutchinson Group, 117-127.

Hall, Stuart. 1997. «The work of representation». En S. Hall (ed.). Representations: Cultural representations and signifying practices. London: Sage, 13-74.

Hart, Chris. 2008. Doing a literature review: Releasing the social science research imagination. London: Sage. 
Hepp, Andreas y Couldry, Nick. 2009. «What should comparative media research be comparing? Towards a transcultural approach to 'media cultures'». En D. Thussu (ed.). Internationalizing media studies. Abingdon: Routledge, 32-48.

Jensen, Klaus. 2015. La comunicación y los medios: metodologías de investigación cualitativa y cuantitativa. México: Fondo de Cultura Económica.

León-Duarte, Guillermo. 2001. «Teorías e investigación de la comunicación en América Latina. Situación actual». Ámbitos, 7(8): 19-47. Disponible en: https://revistas.awpruebas.es/index.php/Ambitos/article/view/9464

León-Duarte, Guillermo. 2007. Sobre la investigación de la comunicación en América Latina. Estrategias y prácticas científicas de la comunicación hoy. Hermosillo: Universidad de Sonora.

Lozano, Carlos. 1996. Teoría e Investigación de la Comunicación de Masas. México: Pearson.

Lozano, Elizabeth. 2006. «Del sujeto cautivo a los consumidores nomádicos». En El consumo cultural en América Latina, ed. Sunkel, Guillermo. Bogotá: Convenio Andrés Bello, 96-113.

Lozano, José y Frankenberg, Lorena. 2008. «Enfoques teóricos y estrategias metodológicas en la investigación empírica de audiencias televisivas en América Latina: 1992-2007». Comunicación y Sociedad, 10: 81-110. Disponible en: http://www.comunicacionysociedad.cucsh.udg.mx/index.php/comsoc/article/view $/ 1844$

Marques de Melo, José. 1998. Teoria da comunicação: paradigmas latinoamericanos. Petrópolis: Vozes.

Marques de Melo, José. 1999. «Paradigmas de escuelas latinoamericanas de comunicación». Revista Latina de Comunicación Social, 19: 1-8. Disponible en: www. revistalatinacs .org/a1999fjl/73meloe.htm.

Marques de Melo, José, Gobbi, María y Kunsch, Waldemar. 2002. Matrizes comunicacionais latinoamericanas: marxismo e cristianismo. São Bernardo do Campo, São Paulo: Unesco-Umesp.

Martín-Barbero, Jesús. 2002. Oficio de Cartógrafo: Travesías latinoamericanas de la comunicación en la cultura. México: FCE.

Martín-Barbero, Jesús. 1978. Comunicación masiva: discurso y poder. Quito: Ciespal.

Martín-Barbero, Jesús. 1987. De los medios a las mediaciones. Comunicación, cultura y hegemonía. Barcelona: Gili.

Martínez-Nicolás, Manuel y Saperas-Lapiedra, Enric. 2016. «Objetos de estudio y orientación metodológica de la reciente investigación sobre comunicación en España (2008-2014)». Revista Latina de Comunicación Social, 71, 1365-1384. doi: 10.4185/RLCS-2016-1150es

Martyniuk, Valdenise y Silva, Simone. 2018. «Between contractual and polemical: Value and representation in marketing narratives». Comunicação, Mídia e Consumo, 14(3): 115-136. doi: 10.18568/cmc.v14i41.1468

Marx, Karl. 1867. Capital: Critique of the political economy. Vol. 1. London: Penguin.

Masotta, Oscar et al. 1967. Happenings. Buenos Aires: Jorge Álvarez.

Mattelart, Armand y Mattelart, Michele. 1997. Historia de las teorías de la comunicación. Barcelona: Paidos.

Mattelart, Armand., Mattelart, Michele y Piccini, Mabel. 1970. «Los medios de comunicación de masas. La ideología de la prensa liberal». Santiago de Chile: Universidad Católica de Chile: Cuadernos de la Realidad Nacional (CEREN), no 3.

Mellado, Claudia. 2010. "Análisis estructural de la investigación empírica sobre el periodista latinoamericano». Comunicación y Sociedad, 13: 125-147. Disponible 
en: $\quad$ http://www.scielo.org.mx/scielo.php?script=sci_abstract\&pid=S0188252X2010000100006\&lng=es\&nrm=iso

Merrigan, Gerianne y Huston, Carole. 2009. Communication research methods. New York City, NY: Oxford University Press.

Mignolo, Walter. 2007. «Delinking». Cultural Studies, 2(2): 449-514. Disponible en: https://www.tandfonline.com/doi/abs/10.1080/09502380601162647

Miike, Yoshitaka. 2010. «An anatomy of Eurocentrism in communication scholarship: The role of Asiacentricity in de-westernizing theory and research». China Media Research, 6(1).

Moragas, Miquel. 2011. Interpretar la comunicación. Estudios sobre medios en América y Europa. Barcelona: Gedisa.

Orozco, Guillermo. 1991. «La audiencia frente a la pantalla. Una exploración del proceso de recepción televisiva». Diálogos de la Comunicación, 30: 54-63.

Orozco, Guillermo. 1994. «Televidencia. Perspectivas para el análisis de los procesos de recepción televisiva». Cuadernos de Comunicación y Prácticas Sociales, 6: 69-88. Disponible en: http://www.publicaciones.cucsh.udg.mx/pperiod/comsoc/pdf/2223_1995/185-187.pdf

Osborne, Peter. 2015. «Problematizing disciplinarity, transdisciplinary problematic». Theory, Culture \& Society, 32(5-6): 3-35. doi:10.1177/0263276415592245.

Parra, Elizabeth y Otazo Hermosilla, Jaime. 2014. «Algunas tendencias de la semiótica en Chile a partir del año 1990». En Proceedings of the 12th World Congress of the International Association for Semiotic Studies (IASS/AIS). Sofia: IASS y NBU, 216-226.

Parra, Elizabeth. (2014). «Eliseo Verón y el desarrollo de la semiótica en Chile». Comunicación y Medios, 29, 129-133. Disponible en: https://revistas.uchile.cl/index.php/RCM/article/ view/32373/34147

Peirce, Charles. 1986. La ciencia de la semiótica. Buenos Aires: Ediciones Nueva Visión.

Pereira, José. 2018. «La investigación en comunicación en Colombia: Síntesis (in)acabada de una travesía». En D. Crovi y R. Trejo (Coords.), Tejiendo nuestra historia. Investigación de la comunicación en América Latina. México D.F.: UNAM, 177-201.

Perelman, Chaim y Olbrechts-Tyteca, Lucie. 1989. Tratado de la argumentación. Madrid: Gredos.

Pessoa de Barros, Diana. 2012. «A semiótica no Brasil e na América do Sul: rumos, papéis e desvios». Revista de Estudos da Linguagem, 20(1): 149-186. doi: 10.17851/2237-2083.20.1.149-186

Piñeiro-Naval, Valeriano y Morais, Ricardo. 2019. «Estudio de la producción académica sobre comunicación en España e Hispanoamérica». Comunicar, 61, 113-123. doi: 10.3916/C61-2019-10.

Piñuel, José y Morales, Enrique. 2018. «Un estudio praxeológico de la investigación en comunicación, a través del análisis del discurso hegemónico de investigadores académicos en España». Perspectivas de la Comunicación, 11(2), 83-135. Disponible en: http://revistas.ufro.cl/ojs/index.php/perspectivas/article/view/1580.

Potter, Jonattan. 1996. La representación de la realidad. Barcelona: Paidos.

Prieto, Luis. 1967. Mensajes y señales. Barcelona: Seix Barral.

Prieto Castillo, Daniel. 1979. Retórica y manipulación masiva. México D.F.: Edicol.

Restrepo, Paula y Valencia, Juan Carlos. 2016. «Prácticas comunicativas en el Buen Vivir». En P. Restrepo, J. Valencia y C. Maldonado (eds.), Comunicación, 
movilizaciones sociales y postdesarrollo: la revolución sí está sucediendo. Quito: Ciespal, 35-60.

Ricoeur, Paul. 1984. Time and narrative. Chicago: University of Chicago Press.

Rodrigo-Mendizábal, Iván (2018). «Análisis del discurso en Latinoamérica: un estado de arte». Chasqui, 139: 15-66. doi: https://doi.org/10.16921/chasqui.v0i139.3934

Romera-Castillo, José (2016). «La revista Signa: 25 años de andadura científica». Signa: Revista de la Asociación Española de Semiótica, 25: 13-76. doi: 10.5944/signa.vol25.2016.16924

Rorty, Richard (Ed.) 1992. The linguistic turn: Essays in philosophical method. Chicago: University of Chicago Press. [(1990). El giro lingüístico. Dificultades metafilosóficas de la filosofia lingüistica. Barcelona: Paidós]

Saussure, Ferdinand de. 1945. Curso de lingüistica general. Buenos Aires: Losada.

Sawyer, R. Keith. 2002. «A discourse on discourse: An archeological history of an intellectual concept». Cultural Studies, 16(3): 433-456. doi: https://doi.org/10.1080/09502380210128324

Scolari, Carlos. 2011. «Introducción. La semiótica en América Latina». Revista LIS Letra Imagen Sonido, 6-7: 13-30. Disponible en: https://dialnet.unirioja.es/servlet/articulo?codigo $=5837830$

Sollaci, Luciana B. y Pereira, Mauricio G. 2004. «The introduction, methods, results, and discussion (IMRAD) structure: a fifty-year survey». Journal of the Medical Library Association, 92(3): 364-367.

https://www.ncbi.nlm.nih.gov/pmc/articles/PMC442179/

Staples, Mike y Niaci, Mahmood. 2007. «Experiences using systematic review guidelines». Journal of Systems and Software, 80(9): 1425-1437. doi: 10.1016/j.jss.2006.09.046

Sunkel, Guillermo. 2006. «El consumo cultural en la investigación en comunicacióncultura en América Latina». En G. Sunkel (comp.), El consumo cultural en América Latina. Bogotá. Convenio Andrés Bello, 15-46.

Torrico, Erik. 2004. Abordajes y periodos de la teoría de la comunicación. Bogotá: Norma.

Tracy, Karen y Haspel, Kathleen. 2004. Language and social interaction: Its institutional identity, intellectual landscape, and discipline-shifting agenda. Journal of Communication, 54(4): 788-816. doi: 10.1093/joc/54.4.788

Vasallo de Lopes, Maria. 2000. «El campo de la Comunicación: reflexiones sobre su estatuto disciplinar». Oficios Terrestres, 7(8): 75-83. Disponible en: http://sedici.unlp.edu.ar/handle/10915/47380

Verón, Eliseo. 1974. «Acerca de la producción social del conocimiento: el 'estructuralismo' y la semiología en Argentina y Chile». Lenguajes, 1: 96-125.

Verón, Eliseo. 1969. Conducta, estructura y comunicación. Buenos Aires: Jorge Álvarez.

Vidales-Gonzáles, Carlos. 2015. «Historia, teoría e investigación de la comunicación». Comunicación y Sociedad, 23: 11-43. Disponible en: http://www.comunicacion ysociedad.cucsh.udg.mx/index.php/comsoc/article/view/60.

Vizcarrondo, Doris. 2017. «Macroproposiciones y legitimación: el debate sobre la ley SB 1070 en la prensa anglosajona e hispana de los Estados Unidos». Comunicação, Mídia e Consumo, 14(41): 84-101. Disponible en: http://revistacmc.espm.br/ index.php/revistacmc/article/view/1578

Wang, Bo. 2004. «A survey of research in Asian rhetoric». Rhetoric Review, 23(2): 171181. Disponible en: https://www.jstor.org/stable/20176611?seq=1 
Zarowsky, Mariano. 2017. Los estudios en comunicación en la Argentina. Ideas, intelectuales, tradiciones politico-culutrales (1956-1985). Buenos Aires: Eudeba.

Zarowsky, Mariano. 2008. «Entre París y Santiago de Chile. Circulación de ideas y redes intelectuales en la recepción de Armand Mattelart de la semiología y la problemática ideológica». Question, 1(18): 1-18. Disponible en: https://perio.unlp.edu.ar/ojs/ index.php/question/article/view/585. 